\title{
Weak and Strong Time Consistency in Differential Oligopoly Games with Capital Accumulation ${ }^{1}$
}

\author{
Roberto Cellini ${ }^{\S}$ - Luca Lambertini*\# \\ $\S$ Department of Economics, University of Catania \\ Corso Italia 55, 95129 Catania, Italy \\ phone 39-095375344, fax 39-095-370574, cellini@unict.it \\ * Department of Economics, University of Bologna \\ Strada Maggiore 45, 40125 Bologna, Italy \\ phone 39-051-2092600, fax 39-051-2092664, lamberti@spbo.unibo.it \\ \# ENCORE, Faculty of Economics \& Econometrics \\ University of Amsterdam, WB 1018 Amsterdam, The Netherlands
}

July 25, 2005

\footnotetext{
${ }^{1}$ We thank George Leitmann and Massimo Marinacci for useful comments and suggestions. The usual disclaimer applies.
} 


\begin{abstract}
We illustrate two differential oligopoly games with capital accumulation where, alternatively, the accumulation dynamics of productive capacity is modelled either à la Solow-Swan or à la Ramsey. We show that in the first case the open-loop Nash equilibrium is only weakly time consistent, while in the second it is strongly so, although the Ramsey game is not state linear.
\end{abstract}

Keywords: differential games, capital accumulation, open-loop equilibria, closed-loop equilibria 


\section{Introduction}

The existing literature on differential games applied to firms' behaviour mainly concentrates on two kinds of strategies adopted by players: the openloop and the closed-loop strategies. ${ }^{1}$ When players (firms) adopt the openloop solution concept, they design the time path concerning the control variable(s) at the initial time and then stick to it forever. This means that the open-loop strategy is simply a time path of actions, and time is the only determinant of the action to be done at any instant. The relevant equilibrium concept is the open-loop Nash equilibrium, which is only weakly time consistent and therefore, in general, it is not subgame perfect. ${ }^{2}$ When players adopt the closed-loop strategy, they do not precommit control variable(s) on any path, and their actions at any instant may depend on all the preceding history. In particular, the values of the state variables are taken into account when players choose their actions at any time. In this situation, the information set used by players in setting their actions at any given instant is often simplified to be only the current value of the state variables at that time, along with the initial conditions. This specific situation is labelled memoryless closed-loop. The relevant equilibrium concept, in this case, is the closed-loop memoryless Nash equilibrium, which is strongly time consistent (or subgame perfect). A different refinement of the closed-loop Nash equilibrium, which is known as the feedback Nash equilibrium, can also be

\footnotetext{
${ }^{1}$ See Kamien and Schwartz (1981); Başar and Olsder (1982, 1995²); Mehlmann (1988); Dockner et al. (2000).

${ }^{2}$ As to the definition of time consistency and subgame perfection, we rely - among many different definitions available in the literature - on the definition provided by Dockner et al. (2000, Section 4.3). We use "strong time consistency" as a synonomous of subgame perfection. Further details are provided below.
} 
adopted as the solution concept. ${ }^{3}$ While in the closed-loop memoryless case the initial and current levels of all state variables are taken into account, in the feedback case only the current stocks of states are considered. ${ }^{4}$

It is worth recalling that "open-loop" and "closed-loop" labels denote different concepts of strategies (in the former, optimal plans depend only on time, whereas in the latter situation, plans depend on the history of the game, possibly summarised by the current stocks of state variables), as well as different kinds of information sets to be used by players (under the open-loop information structure, players' plans associate actions to time only, whereas under the closed-loop information structure, the value of the states has also to be taken into account by players).

The existing literature in this field devotes a considerable amount of attention to identifying classes of games where either the feedback or the closed-loop equilibria degenerate into open-loop equilibria. The degeneration means that the Nash equilibrium time path of the control variables coincide under the different strategy concepts. The interest in the coincidence between the equilibrium path under the different solution concepts is motivated by the following reason. Whenever an open-loop equilibrium is a degenerate closed-loop equilibrium, then the former is also strongly time consistent (or subgame perfect). Therefore, one can rely upon the open-loop equilibrium which, in general, is much easier to derive than closed-loop or feedback ones. Classes of games where this coincidence arises are illustrated in Clemhout and Wan (1974); Reinganum (1982); Mehlmann and Willing (1983); Dockner, Feichtinger and Jørgensen (1985); Fershtman (1987); Fer-

\footnotetext{
${ }^{3}$ For oligopoly models where firms follow feedback rules, see Simaan and Takayama (1978), Fershtman and Kamien (1987, 1990), Dockner and Haug (1990), Cellini and Lambertini (2004), inter alia.

${ }^{4}$ For a clear exposition of the difference among these equilibrium solutions see Başar and Olsder (1982, pp. 318-327, and chapter 6, in particular Proposition 6.1).
} 
shtman, Kamien and Muller (1992). ${ }^{5}$ As a whole, the games where open-loop equilibria are strongly time consistent are known as perfect or state redundant, precisely because optimal controls derived both in open-loop and in closed-loop depend only upon time but not upon states. Hence, open-loop controls are indeed degenerate closed-loop ones. A class of games where this clearly applies is that of linear state games, where the Hamiltonian function is linear in the state variables.

In this paper, we present two classes of Cournot oligopoly games with capital accumulation. In particular, we will consider: (a) the accumulation under the model of reversible investment à la Nerlove-Arrow (1962), where capital accumulation occurs through costly investment, as in Solow's (1956) and Swan's (1956) growth model; (b) the model à la Ramsey (1928), i.e., a "corn-corn" growth model, where accumulation coincides with consumption postponement. Both accumulation mechanisms are widely studied by available theoretical models. We show that only under the Ramsey capital accumulation dynamics, the open-loop Nash equilibrium is a degenerate closed-loop one and hence the former is subgame perfect. This depends on two features: first, the dynamic behaviour of any firm's state variable does not depend on the rivals' control and state variables, which makes the kinematic equations concerning other firms redundant; second, for any firm, the first order conditions taken w.r.t. the control variables are independent of the rivals' state variables, which entails that the cross effect from rivals' states to own controls (which characterises the closed-loop information structure) disappears.

The remainder of the paper is structured as follows. The basics of the

\footnotetext{
${ }^{5}$ For an overview, see Mehlmann (1988) and Dockner et al. (2000, ch. 7). The related issue of time consistency of Stackelberg open-loop equilibria is not treated here (see Cellini, Lambertini and Leitmann, 2005).
} 
model are laid out in section 2. Section 3 examines the two capital accumulation games. Section 4 contains concluding remarks.

\section{The general setup}

The game is played over continuous time, $t \in[0, \infty) .{ }^{6}$ Define the set of players as $\mathbb{P} \equiv\{1,2,3, \ldots N\}$. Moreover, let $x_{i}(t)$ and $u_{i}(t)$ define, as usual, the state variable and the control variable pertaining to player $i$. Assume there exists a prescribed set $\mathcal{U}_{i}$ such that any admissible action $u_{i}(t) \in \mathcal{U}_{i}$. The dynamics of player $i$ 's state variable is described by the following:

$$
\frac{d x_{i}(t)}{d t} \equiv \dot{x}_{i}(t)=f_{i}(\mathbf{x}(t), \mathbf{u}(t))
$$

where $\mathbf{x}(t)=\left(x_{1}(t), x_{2}(t), \ldots x_{N}(t)\right)$ is the vector of state variables at time $t$, and $\mathbf{u}(t)=\left(u_{1}(t), u_{2}(t), \ldots u_{N}(t)\right)$ is the vector of players' actions at the same date, i.e., it is the vector of control variables at time $t$. That is, in the most general case, the dynamics of the state variable associated with player $i$ depends on all state and control variables associated with all players involved in the game. The value of the state variables at $t=0$ is assumed to be known: $\mathbf{x}(0)=\left(x_{1}(0), x_{2}(0), \ldots x_{N}(0)\right)$.

Each player has an objective function, defined as the discounted value of the flow of payoffs over time. The instantaneous payoff depends upon the choices made by player $i$ as well as its rivals, that is:

$$
\pi_{i} \equiv \pi_{i}(\mathbf{x}(t), \mathbf{u}(t))
$$

Player $i$ 's objective is then, for all given $u_{j}(t), j \neq i$ :

$$
\max _{u_{i}} J_{i} \equiv \int_{0}^{\infty} \pi_{i}(\mathbf{x}(t), \mathbf{u}(t)) e^{-\rho t} d t
$$

\footnotetext{
${ }^{6}$ The game can be reformulated in discrete time without significantly affecting its qualitative properties. For further details, see Başar and Olsder $\left(1982,1995^{2}\right)$.
} 
subject to the dynamic constraint represented by the behaviour of the state variables, $(1), u_{i}(t) \in \mathcal{U}_{i}$ and initial conditions $\mathbf{x}(0)=\left(x_{1}(0), x_{2}(0), \ldots x_{N}(0)\right)$. The factor $e^{-\rho t}$ discounts future gains, and the discount rate $\rho$ is assumed to be constant and common to all players. In order to solve his optimisation problem, each player defines a strategy on the basis of a given information structure. We will consider two different information structures, to which two different equilibrium concepts are associated:

Definition 1: open-loop information In this case, each player's information set at any time $t$ includes only calendar time, but neither the history nor the current stocks of states. Hence, the optimal choice of control $u_{i}$ is conditional on current time only, so that the optimal open-loop strategy appears as $u_{i}^{*}=u_{i}^{*}(t)$.

Definition 2: closed-loop memoryless information In this case, each player's information set at any time $t$ consists of the state vector $X(t)$. Hence, the optimal closed-loop memoryless strategy is $u_{i}^{*}=u_{i}^{*}(X(t))$.

By 'memoryless', it is meant that the game history in itself is not relevant for the choice of optimal behaviour at time $t$, only the consequences of the history are important, as they are reflected in the current state vector. However, taking into account the current state vector allows each player to optimally react to the other players' behaviour, because of the effect exerted by the other players' strategies through the vector $X(t)$.

In the literature on differential games, one usually refers to the concepts of weak and strong time consistency. The difference between these two properties can be outlined as follows:

Definition 3: weak time consistency Consider a game played over $t=$ $[0, \infty)$ and examine the trajectories of the state variables, denoted by 
$\mathbf{x}(t)$. The equilibrium is weakly time consistent if its truncated part in the time interval $t=[T, \infty)$, with $T \in(0, \infty)$, represents an equilibrium also for the subgame starting from $t=T$, given the vector of initial conditions $\mathbf{x}_{T}=\mathbf{x}(T)$.

Definition 4: strong time consistency Consider a game played over $t=$ $[0, \infty)$. The equilibrium is strongly time consistent, if its truncated part over $t=[T, \infty)$, with $T \in(0, \infty)$, is an equilibrium for the subgame, independently of the conditions regarding state variables at time $T$, $\mathbf{x}(T)$.

Strong time consistency requires the ability on the part of each player to account for the rival's behaviour at any point in time, i.e., it is, in general, an attribute of closed-loop equilibria, and corresponds to subgame perfection. Weak time consistency is a milder requirement and does not ensure, in general, that the resulting Nash equilibrium be subgame perfect. ${ }^{7}$

In the particular case where the feedback exerted by the other players' strategies on player $i$ 's optimal choice at any $t$ during the game is nil, the resulting optimal closed-loop strategy is $u_{i}^{*}(t)$, i.e., it is no longer a function of $X(t)$. As a result, the closed-loop memoryless strategy collapses into the open-loop one. In the remainder of the section, we clarify under what circumstances the resulting equilibrium $\mathbf{u}^{*}(t) \equiv\left(u_{1}^{*}(t), u_{2}^{*}(t), \ldots u_{N}^{*}(t)\right)$ is indeed strongly time consistent.

\footnotetext{
${ }^{7}$ For a more detailed analysis of these issues, see Dockner, Jørgensen, Long and Sorger (2000, Section 4.3, pp. 98-107); see also Başar and Olsder (1982, 1995², ch. 6).
} 


\subsection{Solution methods}

The Hamiltonian of player $i$ writes as follows: ${ }^{8}$

$$
\begin{aligned}
\mathcal{H}_{i}(\mathbf{x}(t), \mathbf{u}(t)) \equiv & e^{-\rho t}\left[\pi_{i}(\mathbf{x}(t), \mathbf{u}(t))+\lambda_{i i}(t) \cdot f_{i}(\mathbf{x}(t), \mathbf{u}(t))+\right. \\
& \left.+\sum_{j \neq i} \lambda_{i j}(t) \cdot f_{j}(\mathbf{x}(t), \mathbf{u}(t))\right]
\end{aligned}
$$

where $\lambda_{i j}(t)=\mu_{i j}(t) e^{\rho t}$ is the co-state variable (evaluated at time $t$ ) associated with the state variable $x_{j}$.

The first order condition on the control variable $u_{i}(t)$ is:

$$
\frac{\partial \mathcal{H}_{i}(\cdot)}{\partial u_{i}(t)}=0
$$

and the adjoint equations concerning the dynamics of state and co-state variables are as follows:

$$
-\frac{\partial \mathcal{H}_{i}(\cdot)}{\partial x_{j}(t)}=\frac{\partial \lambda_{i j}(t)}{\partial t}-\rho \lambda_{i j}(t), \forall j=1,2 \ldots N
$$

They have to be considered along with the initial conditions $\mathbf{x}(0)$ and the transversality conditions, which set the final value (at $t=\infty$ ) of the state and/or co-state variables:

$$
\lim _{t \rightarrow \infty} e^{-\rho t} \lambda_{i j}(t) x_{j}(t)=0, \forall j=1,2 \ldots N .
$$

From (5) one obtains the instantaneous best reply of player $i$, which can be differentiated with respect to time to yield the kinematic equation of the control variable $u_{i}(t)$. This, in combination with the state equations (1) and the adjoint equations (6) permits to identify the open-loop Nash equilibrium (or equilibria) $)^{9}$ of the game; the optimal time path of the control variables will

\footnotetext{
${ }^{8}$ We assume that regularity and concavity conditions are met.

${ }^{9} \mathrm{We}$ do not deal with the issue of unicity. The properties which we are about to derive apply independently of the number of equilibria.
} 
depend on $t$ only. Moreover, the simultaneous consideration of the dynamic constraint lead to a dynamic system, whose properties can be easily studied.

Under the closed-loop memoryless information structure, the Hamiltonian of player $i$ is the same as in (4). The relevant first order conditions and the adjoint equations are:

$$
\begin{gathered}
\frac{\partial \mathcal{H}_{i}(\cdot)}{\partial u_{i}(t)}=0 ; \\
-\frac{\partial \mathcal{H}_{i}(\cdot)}{\partial x_{j}(t)}-\sum_{h \neq j} \frac{\partial \mathcal{H}_{i}(\cdot)}{\partial u_{h}(t)} \frac{\partial u_{h}^{*}(t)}{\partial x_{j}(t)}=\frac{\partial \lambda_{i j}(t)}{\partial t}-\rho \lambda_{i j}(t), \forall j=1,2 \ldots N ;
\end{gathered}
$$

along with the initial conditions $\mathbf{x}(0)$ and the transversality conditions

$$
\lim _{t \rightarrow \infty} e^{-\rho t} \lambda_{i j}(t) x_{j}(t)=0, \forall j=1,2 \ldots N
$$

The terms

$$
\frac{\partial \mathcal{H}_{i}(\cdot)}{\partial u_{h}(t)} \frac{\partial u_{h}^{*}(t)}{\partial x_{j}(t)}
$$

appearing in the adjoint equations capture the strategic interaction through the feedback from states to controls, which is by definition absent under the open-loop solution concept. In equations $(9)$ and $(11), u_{h}^{*}(t)$ is the solution to the first order condition of firm $h$ w.r.t. her control variable. Whenever the expression in (11) is zero for all $h$, then the closed-loop memoryless equilibrium collapses into the open-loop Nash equilibrium, in the sense that the time path of all relevant variables under the two different information structures coincide. This can happen either because:

$$
\frac{\partial \mathcal{H}_{i}(\cdot)}{\partial u_{h}(t)}=0 \text { for all } h \neq i,
$$

which obtains if the Hamiltonian of player $i$ is a function of his control variable but not of the rivals'; or because:

$$
\frac{\partial u_{h}^{*}(t)}{\partial x_{j}(t)}=0 \text { for all } h \neq j
$$


which means that the first order condition of a player with respect to his control variable does not contain the state variables pertaining to any other players. Of course, it could also be that (12) and (13) hold simultaneously. In particular, $\partial u_{h}^{*}(t) / \partial x_{j}(t)$ may depend upon the solution of the differential equations (9) yielding the expressions for the co-state variables. If the solution of co-state equations:

$$
\lambda_{i j}(t)=\int \frac{\partial \lambda_{i j}(t)}{\partial t} d t
$$

depends only upon time, then indeed (13) holds. Otherwise, if (14) depends upon $x_{h}(t)$, then (13) does not hold. In the first case, the open-loop equilibrium is a degenerate closed-loop one, and both are strongly time consistent (i.e., subgame perfect); in the second case, they are distinct, and the openloop equilibrium is only weakly time consistent.

The property whereby optimal controls are independent of states and initial conditions is known as state-redundancy, and the game itself as stateredundant or perfect (Fershtman, 1987; see also Mehlmann, 1988, ch. 4). From a technical point of view, state-redundancy implies that the control paths are uniquely determined by the co-state trajectories, being unaffected by any feedbacks operating through $X(t)$. In particular, (12) requires the Hamiltonian of player $i$ to be independent of player $h$ 's behaviour, for all $h \neq i$. Intuitively, this holds if and only if there is no strategic interaction at all, i.e., in optimal control problems with a single agent. This of course is no longer a game. On the other hand, (13) applies whenever the Hamiltonian function of player $i$ is additively separable w.r.t. player $i$ 's control and player $j$ 's state, for all $j \neq i$. Intuitively, player $i$ 's payoff is obviously affected by the rivals' states and controls; however, they do not affect his optimal behaviour at any $t$, due to the property of additive separability. It is nevertheless true that additive separability between one's own control(s) and the rival's 
states is sufficient but not necessary in order for the game to be stateredundant. ${ }^{10}$

In the remainder of the analysis, we solve two alternative capital accumulation games according to the closed-loop memoryless concept. In the first case, where the model is studied in a partial equilibrium perspective, we find that (11) does not hold and the open-loop equilibrium is only weakly time consistent. In the second case, which is solved through a general equilibrium approach, (11) holds, so that the closed-loop equilibrium path coincides with the open-loop equilibrium path. As a consequence, the open-loop Nash equilibrium of the second game is not only weakly time consistent, but also strongly so (or subgame perfect).

\section{$3 \quad$ Examples}

We consider two well known market models. In both models, the market exists over $t \in[0, \infty)$, and is served by $N$ firms producing a homogeneous good. Let $q_{i}(t)$ define the quantity sold by firm $i$ at time $t$. The marginal production cost is constant and equal to $c$ for all firms. Firms compete à la Cournot, the demand function at time $t$ being:

$$
p(t)=A-B Q(t), Q(t) \equiv \sum_{i=1}^{N} q_{i}(t) .
$$

In order to produce, firms must accumulate capacity or physical capital $k_{i}(t)$ over time. The two models we consider in the present paper are characterised by two different kinematic equations for capital accumulation.

A] The Nerlove-Arrow (1962) or Solow (1956) - Swan (1956) setting, with

\footnotetext{
${ }^{10}$ Examples of state-redundant games where such separability does not hold are Leitmann and Schmitendorf (1978), Feichtinger (1983) and Cellini and Lambertini (2005).
} 
the relevant dynamic equation being: ${ }^{11}$

$$
\frac{d k_{i}(t)}{d t}=I_{i}(t)-\delta k_{i}(t)
$$

where $I_{i}(t)$ is the investment carried out by firm $i$ at time $t$, and $\delta>0$ is the constant depreciation rate. The instantaneous cost of investment is $C_{i}\left[I_{i}(t)\right]=b\left[I_{i}(t)\right]^{2} / 2$, with $b>0$. We also assume that firms operate with a decreasing returns technology $q_{i}(t)=f\left(k_{i}(t)\right)$, with $f^{\prime} \equiv \partial f\left(k_{i}(t)\right) / \partial k_{i}(t)>0$ and $f^{\prime \prime} \equiv \partial^{2} f\left(k_{i}(t)\right) / \partial k_{i}(t)^{2}<0$. The demand function rewrites as: ${ }^{12}$

$$
p(t)=A-B \sum_{i=1}^{N} f\left(k_{i}(t)\right) .
$$

Here, the control variable is the instantaneous investment $I_{i}(t)$, while the state variable is obviously $k_{i}(t)$. Note that fund raising for financing investment $I_{i}(t)$ is not modelled; hence, this game is solved in partial equilibrium, as the features of the financial market are left out of the picture.

B] The Ramsey (1928) setting, whit the following dynamic equation:

$$
\frac{d k_{i}(t)}{d t}=f\left(k_{i}(t)\right)-q_{i}(t)-\delta k_{i}(t)
$$

\footnotetext{
${ }^{11}$ Traditionally, differential equations as in (16) have been used to describe the dynamics of market shares in advertising models, following the seminal contribution of Nerlove and Arrow (1962). See Feichtinger, Hartl and Sethi (1994). However, the same dynamics also describes capital accumulation in Solow (1956) and Swan (1956).

${ }^{12}$ Notice that the assumption $q_{i}(t)=f\left(k_{i}(t)\right)$ entails that firms always operate at full capacity. This, in turn, amounts to saying that this model encompasses the case of Bertrand behaviour under capacity constraints, as in Kreps and Scheinkman (1983), inter alia. The open-loop solution of the Nerlove-Arrow differential game in a duopoly model is in Fershtman and Muller (1984) and Reynolds (1987). The latter author also derives the feedback solution through Bellman's value function approach.
} 
where $f\left(k_{i}(t)\right)=y_{i}(t)$ denotes the output produced by firm $i$ at time t. Also in this setting, we assume $f^{\prime} \equiv \partial f\left(k_{i}(t)\right) / \partial k_{i}(t)>0$ and $f^{\prime \prime} \equiv \partial^{2} f\left(k_{i}(t)\right) / \partial k_{i}(t)^{2}<0$. In this case, capital accumulates as a result of intertemporal relocation of unsold output $y_{i}(t)-q_{i}(t)$. This can be interpreted in two ways. The first consists in viewing this setup as a corn-corn model, where unsold output is reintroduced in the production process. The second consists in thinking of a two-sector economy where there exists an industry producing the capital input which can be traded against the final good at a fixed price equal to one. In either case, the Ramsey approach is based upon a general equilibrium analysis. In this model, the control variable is $q_{i}(t)$, and the state variable is $k_{i}(t)$.

\subsection{The Nerlove-Arrow or Solow-Swan model}

When capital accumulates according to (16), the relevant Hamiltonian for firm $i$ is:

$$
\begin{aligned}
\mathcal{H}_{i}(\cdot)= & e^{-\rho t}\left\{\left[A-B f\left(k_{i}(t)\right)-B \sum_{j \neq i} f\left(k_{j}(t)\right)-c\right] f\left(k_{i}(t)\right)-\frac{b}{2}\left[I_{i}(t)\right]^{2}+\right. \\
& \left.+\lambda_{i i}(t)\left[I_{i}(t)-\delta k_{i}(t)\right]+\sum_{j \neq i} \lambda_{i j}(t)\left[I_{j}(t)-\delta k_{j}(t)\right]\right\} .
\end{aligned}
$$

Necessary conditions for the closed-loop memoryless equilibrium are: ${ }^{13}$

\footnotetext{
${ }^{13}$ Given that the Hamiltonian is concave, we focus on first order conditions alone. In (20), the indication of exponential discounting is omitted for brevity.
} 


$$
\begin{aligned}
& \text { (i) } \frac{\partial \mathcal{H}_{i}(\cdot)}{\partial I_{i}(t)}=0 \Rightarrow-b I_{i}(t)+\lambda_{i i}(t)=0 \Rightarrow I_{i}^{*}(t)=\lambda_{i i}(t) / b \\
& (i i)-\frac{\partial \mathcal{H}_{i}(\cdot)}{\partial k_{i}(t)}-\sum_{j \neq i} \frac{\partial \mathcal{H}_{i}(t)}{\partial I_{j}(t)} \frac{\partial I_{j}^{*}(t)}{\partial k_{i}(t)}=\frac{\partial \lambda_{i i}(t)}{\partial t}-\rho \lambda_{i i}(t) \Rightarrow \\
& \Rightarrow \frac{\partial \lambda_{i i}(t)}{\partial t}=(\rho+\delta) \lambda_{i i}(t)-\sum_{j \neq i} \frac{\partial \mathcal{H}_{i}(t)}{\partial I_{j}(t)} \frac{\partial I_{j}^{*}(t)}{\partial k_{i}(t)}+ \\
& +f^{\prime}\left(k_{i}(t)\right)\left[2 B f\left(k_{i}(t)\right)+B \sum_{j \neq i} f\left(k_{j}(t)\right)-(A-c)\right] \\
& \left(i i^{\prime}\right)-\frac{\partial \mathcal{H}_{i}(\cdot)}{\partial k_{j}(t)}-\sum_{h \neq j} \frac{\partial \mathcal{H}_{i}(t)}{\partial I_{h}(t)} \frac{\partial I_{h}^{*}(t)}{\partial k_{j}(t)}=\frac{\partial \lambda_{i j}(t)}{\partial t}-\rho \lambda_{i j}(t),
\end{aligned}
$$

with the transversality conditions:

$$
\lim _{t \rightarrow \infty} \mu_{i j}(t) \cdot k_{i}(t)=0 \text { for all } i, j .
$$

Condition $\left(20-i i^{\prime}\right)$, which yields $\partial \lambda_{i j}(t) / \partial t$, is redundant in that $\lambda_{i j}(t)$ does not appear in the first order conditions $(20-i)$ and (20-ii). Moreover, on the basis of $(20-i)$, one could be lead to think that $\partial I_{i}^{*}(t) / \partial k_{i}(t)=0$ for all $i, j$. Yet, this is not the case in view of the co-state equation (20-ii) yielding $\partial \lambda_{i i}(t) / \partial t$ as a function of the whole vector of capacities of firm $i$ as well as the rivals'. Hence, integrating (20-ii) one obtains the expression of $\lambda_{i i}(t)$ which depends on all $k_{j}(t)$. The ultimate consequence of this fact is that the optimal control of firm $i$ is not independent of the state variables of the rivals.

This discussion is summarised by:

Proposition 1 The open-loop solution of the Nerlove-Arrow Solow-Swan game is weakly time consistent.

Therefore, in order to obtain a subgame perfect equilibrium, one has to solve the closed-loop game. This is done, for the duopoly case, by Reynolds (1987). 


\subsection{The Ramsey model}

Under the dynamic constraint (18), the Hamiltonian of firm $i$ is: ${ }^{14}$

$$
\begin{aligned}
\mathcal{H}_{i}(\cdot)= & \left\{e^{-\rho t}\left[A-B q_{i}(t)-B Q_{-i}(t)-c\right] q_{i}(t)+\right. \\
& +\lambda_{i i}(t)\left[f\left(k_{i}(t)\right)-q_{i}(t)-\delta k_{i}(t)\right]+ \\
& \left.+\sum_{j \neq i} \lambda_{i j}(t)\left[f\left(k_{j}(t)\right)-q_{j}(t)-\delta k_{j}(t)\right]\right\}
\end{aligned}
$$

where $Q_{-i}(t)=\sum_{j \neq i} q_{j}(t)$.

The first order condition concerning the control variable is: ${ }^{15}$

$$
\frac{\partial \mathcal{H}_{i}(\cdot)}{\partial q_{i}(t)}=A-2 B q_{i}(t)-B Q_{-i}(t)-c-\lambda_{i i}(t)=0 .
$$

Now examine at the co-state equation of firm $i$ calculated for the state variable of firm $i$ herself, for the closed-loop solution of the game:

$$
\begin{aligned}
-\frac{\partial \mathcal{H}_{i}(\cdot)}{\partial k_{i}(t)}-\sum_{j \neq i} \frac{\partial \mathcal{H}_{i}(t)}{\partial q_{j}(t)} \frac{\partial q_{j}^{*}(t)}{\partial k_{i}(t)} & =\frac{\partial \lambda_{i i}(t)}{\partial t}-\rho \lambda_{i i}(t) \Rightarrow \\
\frac{\partial \lambda_{i i}(t)}{\partial t} & =\lambda_{i i}(t)\left[\rho+\delta-f^{\prime}\left(k_{i}(t)\right)\right],
\end{aligned}
$$

and the co-state equation pertaining to any of the rivals' state variable:

$$
\begin{aligned}
-\frac{\partial \mathcal{H}_{i}(\cdot)}{\partial k_{j}(t)}-\sum_{j \neq h} \frac{\partial \mathcal{H}_{i}(t)}{\partial q_{j}(t)} \frac{\partial q_{h}^{*}(t)}{\partial k_{i}(t)} & =\frac{\partial \lambda_{i j}(t)}{\partial t}-\rho \lambda_{i j}(t) \Rightarrow \\
\frac{\partial \lambda_{i j}(t)}{\partial t} & =\lambda_{i j}(t)\left[\rho+\delta-f^{\prime}\left(k_{j}(t)\right)\right],
\end{aligned}
$$

Note that (i) equation (25) is independent of any $k_{j}(t)$ for all $j \neq i$; and (ii) equation (26) is a separable differential equation admitting the solution $\lambda_{i j}(t)=0$ at any point in time. These two observations involve that

$$
\frac{\partial q_{i}^{*}(t)}{\partial k_{j}(t)}=0 \text { for all } i \neq j
$$

\footnotetext{
${ }^{14} \mathrm{As}$ in the previous section, the Hamiltonian is concave. Therefore, we confine our attention to first order conditions.

${ }^{15}$ Again, exponential discounting is omitted for the sake of brevity.
} 
and the adjoint equations (26) are indeed redundant since $\lambda_{i j}(t)$ does not appear in firm $i$ 's first order condition (24) on the control variable. This amounts to saying that, in the Ramsey game, the open-loop solution is a degenerate closed-loop solution because the best reply function of firm $i$ does not contain the state variables pertaining to any of her rivals. Therefore, we have proved:

Proposition 2 Under the Ramsey capital accumulation dynamics, the closedloop memoryless equilibrium coincides with the open-loop equilibrium, which therefore is strongly time consistent.

Then, using the dynamic constraint (18) and the reaction function (??), it is possible to find the analytical steady state solutions:

$$
q^{S S}=\frac{A-c}{B(N+1)} ; f^{\prime}(k)=\rho+\delta
$$

and to study their dynamic properties. This model can be easily extended to more general cases accounting for product differentiation or non-linear market demand, under either Cournot or Bertrand competition (see Cellini and Lambertini, 1998, 2000, where one can also find the stability analysis).

The interest of the foregoing discussion lies in the possibility of using the open-loop information structure to yield subgame perfect equilibria in a game whose Hamiltonian function is not linear in the state variables. ${ }^{16}$ Therefore, the present game is state redundant although not state linear. This feature makes it possible to extend the above model to investigate, e.g., fiscal or trade policy issues (Baldini and Lambertini, 2002; Calzolari and Lambertini, $2002,2003)$ in a general equilibrium framework.

\footnotetext{
${ }^{16} \mathrm{As}$ is well known, linear state games have the property that their open-loop Nash equilibria are subgame perfect (see Mehlmann, 1988, ch. 4; and Dockner et al., 2000, ch. $7.2)$.
} 


\section{Conclusions}

It is well known that the explicit solution of differential games with closedloop information structures is, in general, cumbersome and often analytically impossible. For this reason, several contributions in the existing literature confine to the characterisation of open-loop equilibria, even if the limitations of the open-loop decision rules are well known, and - in particular - open-loop equilibria are only weakly time consistent.

In the foregoing analysis, we have shown that games of capital accumulation represent a field where the differential game approach can be easily applied. As we have shown, the weak or strong time consistency of openloop Nash equilibria drastically depends upon the way one models the capital accumulation process. Specifically, the capital accumulation rule à la Nerlove-Arrow (or Solow-Swan) gives rise to a weakly time consistent openloop equilibrium, while the Ramsey capital accumulation rule yields strong time consistency. In the latter case, this happens because the first order conditions of any given firm does not contain the state variables pertaining to other firms, even if strategic interdependence in the market phase is indeed present. Hence, this setting can be usefully applied along several directions using the open-loop approach, being the resulting equilibria subgame perfect. 


\section{References}

[1] Baldini, M. and L. Lambertini (2002), "Profit Taxation and Capital Accumulation in Dynamic Oligopoly Models", working paper no. 435, Department of Economics, University of Bologna.

[2] Başar, T., and Olsder, G.J. (1982, 1995²), Dynamic Noncooperative Game Theory, San Diego, Academic Press.

[3] Calzolari, G. and L. Lambertini (2002), "Export Restraints in a Model of Trade with Capital Accumulation", in L.A. Petrosjan and N.A. Zenkevich (eds.), Proceedings of the $X$ International Symposium on Dynamic Games and Applications, International Society of Dynamic Games and St. Petersburg State University, vol. I, 158-71.

[4] Calzolari, G. and L. Lambertini (2003), "Tariffs vs Quotas in a Model of Trade with Capital Accumulation", in R. Neck (ed.), Proceedings of the IFAC Symposium on Modelling and Control of Economic Systems (SME 2001), Oxford, Pergamon Press, 69-74.

[5] Cellini, R. and L. Lambertini (1998), "A Dynamic Model of Differentiated Oligopoly with Capital Accumulation", Journal of Economic Theory, 83, 145-55.

[6] Cellini, R. and L. Lambertini (2000), "Non-Linear Market Demand and Capital Accumulation in a Differential Oligopoly Game", working paper no. 370, Department of Economics, University of Bologna.

[7] Cellini, R. and L. Lambertini (2004), "Dynamic Oligopoly with Sticky Prices: Closed-Loop, Feedback and Open-Loop Solutions", Journal of Dynamical and Control Systems, 10, 303-14. 
[8] Cellini, R. and L. Lambertini (2005), "R\&D Incentives and Market Structure: A Dynamic Analysis", Journal of Optimization Theory and Applications, 126, 85-96.

[9] Cellini, R., L. Lambertini and G. Leitmann (2005), "Degenerate Feedback and Time Consistency in Differential Games", in E.P. Hofer and E. Reithmeier (eds), Modeling and Control of Autonomous Decision Support Based Systems. Proceedings of the 13th Workshop on Dynamics \& Control, Aachen, Shaker Verlag, 185-92.

[10] Clemhout, S. and H.Y. Wan, Jr. (1974), "A Class of Trilinear Differential Games", Journal of Optimization Theory and Applications, 14, 419-24.

[11] Dockner, E.J., G. Feichtinger and S. Jørgensen (1985), "Tractable Classes of Nonzero-Sum Open-Loop Nash Differential Games: Theory and Examples", Journal of Optimization Theory and Applications, 45, 179-97.

[12] Dockner, E.J. and A.A. Haug (1990), "Tariffs and Quotas under Dynamic Duopolistic Competition", Journal of International Economics, 29, 147-59.

[13] Dockner, E.J, S. Jørgensen, N.V. Long and G. Sorger (2000), Differential Games in Economics and Management Science, Cambridge, Cambridge University Press.

[14] Feichtinger, G. (1983), "The Nash Solution of an Advertising Differential Game: Generalization of a Model by Leitmann and Schmitendorf", IEEE Transactions on Automatic Control, 28, 1044-48. 
[15] Feichtinger, G., R.F. Hartl and P.S. Sethi (1994), "Dynamic Optimal Control Models in Advertising: Recent Developments", Management Science, 40, 195-226.

[16] Fershtman, C. (1987), "Identification of Classes of Differential Games for Which the Open-Loop is a Degenerate Feedback Nash Equilibrium", Journal of Optimization Theory and Applications, 55, 217-31.

[17] Fershtman, C. and M.I. Kamien (1987), "Dynamic Duopolistic Competition with Sticky Prices", Econometrica, 55, 1151-64.

[18] Fershtman, C. and M.I. Kamien (1990), "Turnpike Properties in a Finite-Horizon Differential Game: Dynamic Duopoly with Sticky Prices", International Economic Review, 31, 49-60.

[19] Fershtman, C. and E. Muller (1984), "Capital Accumulation Games of Infinite Duration", Journal of Economic Theory, 33, 322-39.

[20] Fershtman, C., M. Kamien and E. Muller (1992), "Integral Games: Theory and Applications", in Feichtinger, G. (ed.), Dynamic Economic Models and Optimal Control, Amsterdam, North-Holland, 297-311.

[21] Kamien, M.I. and N.L. Schwartz (1981), Dynamic optimization: The Calculus of Variations and Optimal Control in Economics and Management, Amsterdam, North-Holland.

[22] Kreps, D. and J. Scheinkman (1983), "Quantity Precommitment and Bertrand Competition Yield Cournot Outcomes", Bell Journal of Economics, 14, 326-37.

[23] Leitmann, G. and W.E. Schmitendorf (1978), "Profit Maximization through Advertising: A Nonzero Sum Differential Game Approach", IEEE Transactions on Automatic Control, 23, 646-50. 
[24] Mehlmann, A. (1988), Applied Differential Games, New York, Plenum Press.

[25] Mehlmann, A. and R. Willing (1983), "On Nonunique Closed-Loop Nash Equilibria for a Class of Differential Games with a Unique and Degenerate Feedback Solution", Journal of Optimization Theory and Applications, 41, 463-72.

[26] Nerlove, M. and K.J. Arrow (1962), "Optimal Advertising Policy under Dynamic Conditions", Economica, 29, 129-42.

[27] Ramsey, F.P. (1928), "A Mathematical Theory of Saving", Economic Journal, 38, 543-59.

[28] Reinganum, J. (1982), "A Class of Differential Games for Which the Closed Loop and Open Loop Nash Equilibria Coincide", Journal of Optimization Theory and Applications, 36, 253-62.

[29] Reynolds, S.S. (1987), "Capacity Investment, Preemption and Commitment in an Infinite Horizon Model", International Economic Review, 28, 69-88.

[30] Simaan, M. and T. Takayama (1978), "Game Theory Applied to Dynamic Duopoly Problems with Production Constraints", Automatica, 14, 161-66.

[31] Solow, R. (1956), "A Contribution to the Theory of Economic Growth", Quarterly Journal of Economics, 70, 65-94.

[32] Swan, T.W. (1956), "Economic Growth and Capital Accumulation", Economic Record, 32, 334-61. 